01

\title{
Конкуренция концентрационного сужения и полевого уширения темного резонанса в лестничной системе уровней атомов рубидия: особенности проявления в тонких спектроскопических ячейках
}

\author{
(C) А. Саргсян ${ }^{1}$, Ч.С. Адамс ${ }^{2}$, Т.А. Вартанян ${ }^{3, \uparrow}$, Д. Саркисян ${ }^{1}$ \\ ${ }^{1}$ Институт фризических исследований НАН Армении, \\ 0203 Аштарак, Армения \\ ${ }^{2}$ Joint Quantum Centre (Durham-Newcastle), Department of Physics, Durham University, South Road, Durham, \\ DH1 3LE, United Kingdom \\ ${ }^{3}$ Университет ИТМО, \\ 197101 Санкт-Петербург, Россия \\ ฯ e-mail: Tigran.Vartanyan@mail.ru
}

Поступила в редакцию 03.06.2020 г.

В окончательной редакции 03.06.2020 г.

Принята к публикации 23.06.2020 г.

Исследован эффект электромагнитно-индуцированной прозрачности в лестничной системе уровней $5 S_{1 / 2}-5 P_{3 / 2}-5 D_{5 / 2}$ атомов $\mathrm{Rb}$. Продемонстрирован эффект спектрального сужения темного резонанса (dark resonance - DR) в зависимости от плотности паров атомов и толщины $L$ спектроскопической ячейки, в которой находились пары атомов $\mathrm{Rb}$. Толщина $L$ варьировалась в интервале от $390 \mathrm{~nm}$ до $4 \mathrm{~mm}$, плотность атомов $N$ увеличивалась до $\sim 10^{16} \mathrm{~cm}^{-3}$, при этом использовалось интенсивное связывающее и слабое пробное излучения. Максимальный эффект, а именно 22-кратное спектральное сужение DR, достигалось в ячейке с толщиной $L=4 \mathrm{~mm}$. С уменьшением толщины $L$ эффект спектрального сужения становится слабее: так, при $L=2 \mu \mathrm{m}$ происходило 2.4-кратное спектральное сужение DR. Спектральное сужение DR практически отсутствовало при $L=0.8 \mu \mathrm{m}$, а при дальнейшем уменьшении до $L=0.4 \mu \mathrm{m}$ с увеличением плотности паров атомов начинало происходить спектральное уширение DR. Практически во всех случаях при умеренных плотностях атомов и больших интенсивностях связывающего излучения достигался $\sim 100 \%$ контраст DR. Приведено объяснение эффекта спектрального сужения и уширения DR.

Ключевые слова: электромагнитно-индуцированная прозрачность, лестничная система уровней, темные резонансы, атомы Rb, допплеровское уширение.

DOI: $10.21883 /$ OS.2020.10.50011.166-20

\section{1. Введение}

К важной особенности эффекта электромагнитноиндуцированной прозрачности (ЭИП) [1-4] следует отнести возможность формирования спектрально-узкого темного оптического резонанса (DR - dark resonance) как в $\Lambda$-системе уровней, так и в лестничной системе уровней ( $\Xi$-система) даже при малых толщинах столба атомарных паров $L \sim 1 \mu \mathrm{m}$ [5]. Это важно для ряда практических приложений, поскольку позволяет не только сильно уменьшить размеры используемых атомарных ячеек для формирования DR [6,7], но и, используя короткофокусные линзы, значительно увеличить интенсивность лазерного излучения. Возможность острой фокусировки приобретает особое значение при использовании высоколежащих ридберговских уровней, когда вероятность второго (верхнего) перехода мала $[8,9]$, и при двухфотонном поглощении [10].

Для формирования DR, как правило, используют $\Lambda$ систему, которая состоит из двух долгоживущих нижних уровней (начального и конечного) и одного высоколежащего уровня. Это позволяет, при определенных условиях, формировать сверхузкие (с шириной в несколько $\mathrm{Hz}$ ) темные оптические резонансы DR [11,12]. Формирование настолько узких резонансов в лестничной $\Xi$ системе в парах атомов невозможно из-за относительно малого времени жизни конечного (высоколежащего) уровня. На рис. 1 показана $\Xi$-система уровней атомa $\mathrm{Rb}, 5 S_{1 / 2}-5 P_{3 / 2}-5 D_{5 / 2}$, у которого естественная ширина конечного уровня $5 D_{5 / 2}$ составляет $0.67 \mathrm{MHz}[13]$. В случае $\Xi$-системы заметный вклад вносит также остаточная допплеровская ширина, обусловленная различием длин волн связывающего $\lambda_{C} \approx 776 \mathrm{~nm}$ и пробного $\lambda_{P} \approx 780 \mathrm{~nm}$ лазеров: $\delta D \approx\left(k_{C}-k_{P}\right) V \approx 2 \mathrm{MHz}$, где $k_{C}=2 \pi / \lambda_{C}, k_{P}=2 \pi / \lambda_{P}, V-$ тепловая скорость атома $\mathrm{Rb}$. Отметим, что указанная ширина достигается только при встречном распространении связывающего и пробного излучения, а при коллинеарном распространении величина $\delta D$ оказывается более чем на 2 порядка больше [14]. Тем не менее DR в $\Xi$-системе $5 S-5 P-n D$ имеет важные практические применения: в работе [8] были измерены величины тонкого расщепления ридберговских уровней атомов $\mathrm{Rb}$ с главными квантовыми числами $n$ от 26 до 124. В работе [10] было показано, что вплоть до толщин $0.7 \mathrm{~mm}$ параметры DR для 


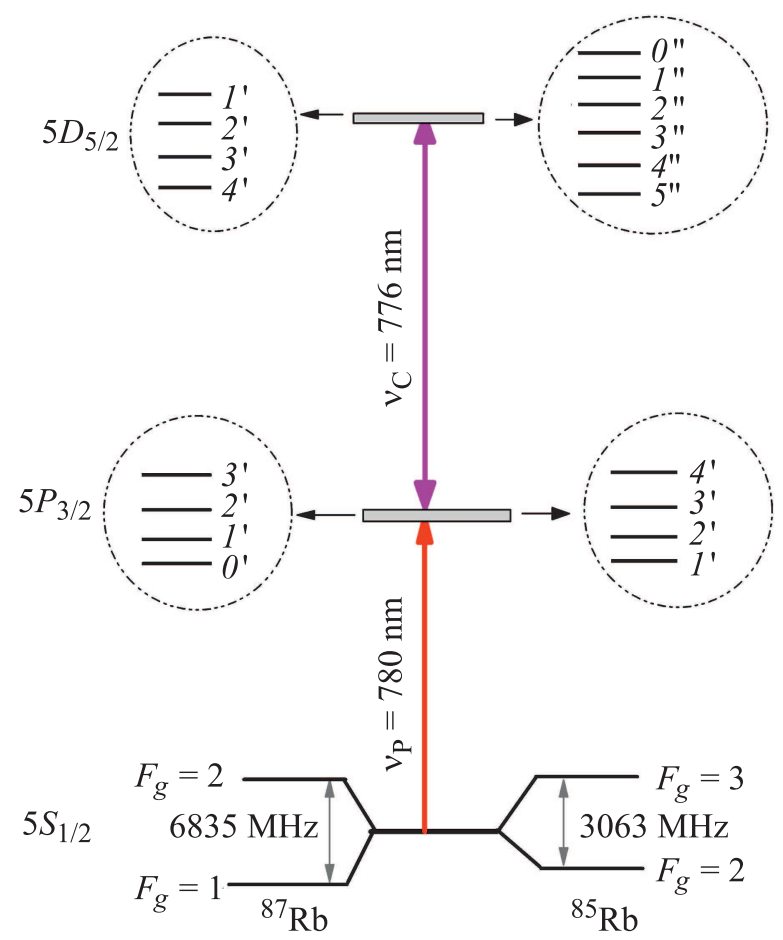

Pис. 1. Диаграмма $\Xi$-системы уровней атомов $\mathrm{Rb}$ $5 S_{1 / 2}-5 P_{3 / 2}-5 D_{5 / 2}$. В кружках показана сверхтонкая структура уровней $5 P_{3 / 2}$ и $5 D_{5 / 2}$ : на левой и правой частях для атомов ${ }^{87} \mathrm{Rb}$ и ${ }^{85} \mathrm{Rb}$ соответственно.

$n=5,26,27$ ухудшаются незначительно, а при наличии двухфотонной расстройки уверенно регистрируется двухфотонное поглощение.

Приведенная на рис. 1 छ-система исследовалась во многих работах, что объясняется наличием хороших узкополосных лазеров на отмеченных выше длинах волн и сравнительно малой, в несколько $\mathrm{MHz}$, шириной DR $[15,16]$. В настоящей работе исследовано влияние плотности паров $\mathrm{Rb}$ и толщины ячейки, в которой они заключены, на спектральную ширину DR.

\section{2. Эксперимент}

\section{1. Конструкции оптических ячеек}

Для изучения влияния толщины столба паров на спектральную ширину темного резонанса были использованы две ячейки. 1-я оптическая ячейка имеет $T$ образную конструкцию и состоит из цилиндрической трубки, изготовленной из кристаллического сапфира $\mathrm{Al}_{2} \mathrm{O}_{3}$, с внутренним диаметром $11 \mathrm{~mm}$ и длиной $4 \mathrm{~mm}$, на торцы которой приклеены хорошо отполированные сапфировые окна толщиной $0.5 \mathrm{~mm}$. В центре цилиндрической трубки проделано небольшое отверстие и приклеен боковой отросток - сапфировая трубка диаметром $2 \mathrm{~mm}$ и длиной $50 \mathrm{~mm}$, которая является резервуаром и заполняется природным Rb, состоящим из изотопов ${ }^{85} \mathrm{Rb}(72 \%)$ и ${ }^{87} \mathrm{Rb}(28 \%)$. Использовался двухсекционный нагреватель, что позволяло нагревать раздельно цилиндрическую трубку с окнами и боковой отросток - резервуар. Температура резервуара, которая определяет плотность атомов рубидия $N$, могла варьироваться в пределах $50-450^{\circ} \mathrm{C}$; на окнах температура поддерживалась на $20-30^{\circ} \mathrm{C}$ выше, чтобы предотвратить конденсацию паров. Более подробное описание конструкции ячейки дано в [10].

Конструкция 2-й, микронной, ячейки с толщиной, варьируемой в интервале $100 \mathrm{~nm}-3 \mu \mathrm{m}$, аналогична той, что описана в $[10,17,18]$. Прямоугольные окна размерами $20 \times 30 \mathrm{~mm}$ и толщиной $2 \mathrm{~mm}$ были изготовлены из отполированного кристаллического сапфира. Кристаллическая ось $C$ была ориентирована перпендикулярно поверхности окна, чтобы уменьшить влияние двулучепреломления. Для формирования клиновидного зазора в нижней части ячейки между окнами помещалась платиновая полоска толщиной $3 \mu \mathrm{m}$. Температурные режимы были такими же, как и в 1-й ячейке.

\section{2. Экспериментальная установка}

Схема экспериментальной установки приведена на рис. 2. Использовалось излучение двух непрерывных узкополосных (с шириной $\sim 1 \mathrm{MHz}$ ) диодных лазеров с внешним резонатором, один из которых - связывающий лазер (coupling laser) - имел фиксированную частоту $v_{C}$, а второй, с перестраиваемой частотой $v_{P}$, являлся пробным (probe laser) лазером. Линейные поляризации связывающего и пробного лазеров были взаимно перпендикулярны. Оба излучения вначале проходят через оптоволокно и линзу, служащую для увеличения диаметра пучков до $1.5 \mathrm{~cm}$ и для обеспечения возможности последующей жесткой фокусировки, и направляются навстречу друг другу. Оба излучения фокусируются линзами с фокусным расстоянием $20 \mathrm{~cm}$ в пятна размером $d \approx 30 \mu \mathrm{m}$ и тщательно пространственно совмещаются в

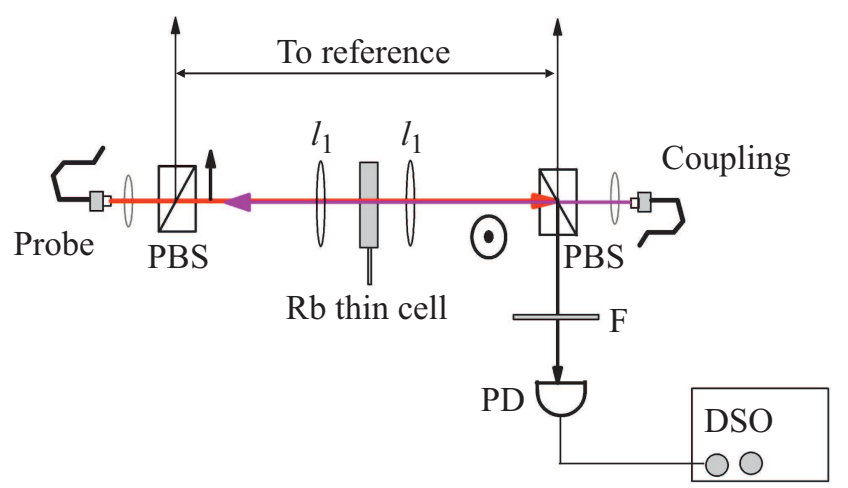

Рис. 2. Схема экспериментальной установки. Пробное $(\lambda \approx 780 \mathrm{~nm})$ и связывающее излучения $(\lambda \approx 776 \mathrm{~nm})$ фокусируются и совмещаются линзами $l_{1}$ в ячейке с парами $\mathrm{Rb}$; $\mathrm{PBS}$ - поляризационная призма; с помощью фотодетектора (PD) регистрируются спектры пробного излучения и частотного репера (reference). Сигналы с PD подаются на цифровой осциллограф (DSO) F-фильтр (Semrock LL01-780-12.5). 
ячейке с парами атомов рубидия. При пролете атома $\mathrm{Rb}$ с тепловой скоростью $V=2 \cdot 10^{4} \mathrm{~cm} / \mathrm{s}$ через пучок диаметром $30 \mu \mathrm{m}$, вследствие конечности времени пролета $T=d / V$, DR резонанс приобретает времяпролетное уширение $\gamma_{T}=1 / 2 \pi T \approx 2 \mathrm{MHz}$. Реперный (reference) спектр формировался известным методом насыщенного поглощения (НП) [19].

\section{3. Экспериментальные результаты}

\subsection{1. Толщина столба паров $L=4 \mathrm{~mm}$}

Отметим работу [20], в которой была использована ячейка длиною $L=4 \mathrm{~mm}$ с парами атомов $\mathrm{Rb}$ и было обнаружено два интересных эффекта: 1) пропускание пробного излучения в результате эффекта ЭИП на частоте перехода $5 S_{1 / 2}-5 P_{3 / 2}$ при определенных параметрах эксперимента может достигать $300 \%$, т.е. имеет место усиление (или генерация) на частоте перехода $5 S_{1 / 2}-5 P_{3 / 2}$; 2) с увеличением плотности атомарных паров может иметь место значительное сужение спектральной ширины DR. В настоящей работе были продолжены исследования DR резонанса в $\Xi$-системе, показанной на диаграмме рис. 1. В эксперименте регистрируется спектр пропускания пробной волны; для выделения частоты $v_{P}$ $(780 \mathrm{~nm})$ используется узкополосный фильтр (Semrock LL01-780-12.5), сильно подавляющий излучение на частоте $v_{C}(776 \mathrm{~nm})$. Частота пробного излучения $v_{P}$ сканируется по переходам $F_{g}=3 \rightarrow F_{e}=2^{\prime}, 3^{\prime}, 4^{\prime} \quad D_{2^{-}}$ линии атома ${ }^{85} \mathrm{Rb}$, а частота $v_{C}$ находится в резонансе с переходом $5 P_{3 / 2}-5 D_{5 / 2} \quad\left(F_{e}=4^{\prime} \rightarrow F_{e}=3^{\prime \prime}, 4^{\prime \prime}, 5^{\prime \prime}\right)$. Верхняя кривая 1 на рис. $3, a$ показывает спектр пробной волны, который содержит DR при температуре ячейки $85^{\circ} \mathrm{C}$ (плотность атомов $N_{\mathrm{Rb}} \sim 2 \cdot 10^{12} \mathrm{~cm}^{-3}$ ), мощности пробной и связывающей волн составляли $6 \mu \mathrm{W}$ и $250 \mathrm{~mW}$ соответственно. Спектральная ширина DR на полувысоте $\sim 166 \mathrm{MHz}$. Контраст DR, который определяется как отношение амплитуды DR к пиковой величине поглощения, $120 \%$ (пиковая величина поглощения столба паров атомов $100 \%$ отмечена на рисунке). Средняя кривая 2 показывает спектр пропускания пробной волны, который содержит DR при температуре ячейки $145^{\circ} \mathrm{C}$ (плотность атомов $N_{\mathrm{Rb}} \sim 6 \cdot 10^{13} \mathrm{~cm}^{-3}$ ). Спектральная ширина DR, которая аппроксимирована лоренцевой кривой, составляет на полувысоте $7.5 \mathrm{MHz}$ (показано на вставке), контраст DR 4\%. Таким образом, несмотря на ухудшение контраста DR, происходит 22кратное сужение спектра DR. На рис. $3, b$ показан спектр пробной волны, который содержит DR при температуре ячейки $90^{\circ} \mathrm{C}\left(N_{\mathrm{Rb}} \sim 2.5 \cdot 10^{12} \mathrm{~cm}^{-3}\right)$, мощности пробной и связывающей волн составляли $1 \mu \mathrm{W}$ и $4 \mathrm{~mW}$ соответственно. Контраст DR в этом случае $13 \%$. На вставке показаны аппроксимированные гауссовыми кривыми переходы $5 P_{3 / 2}-5 D_{5 / 2}\left(F_{g}=2,3,4^{\prime} \rightarrow F_{e}=2^{\prime \prime}, 3^{\prime \prime}, 4^{\prime \prime}, 5^{\prime \prime}\right)$. Разница в функциях, аппроксимирующих DR, обусловлена различием экспериментальных условий при их

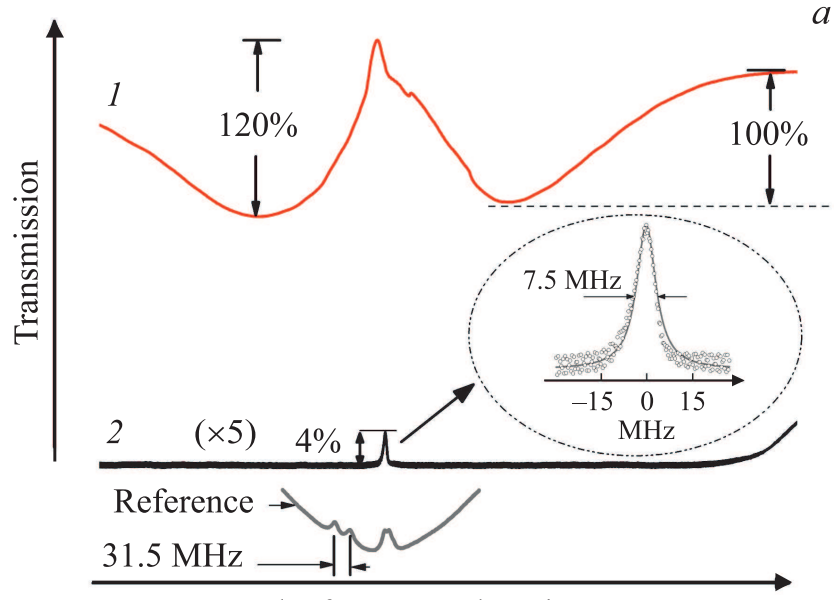

Probe frequency detuning, $\mathrm{MHz}$

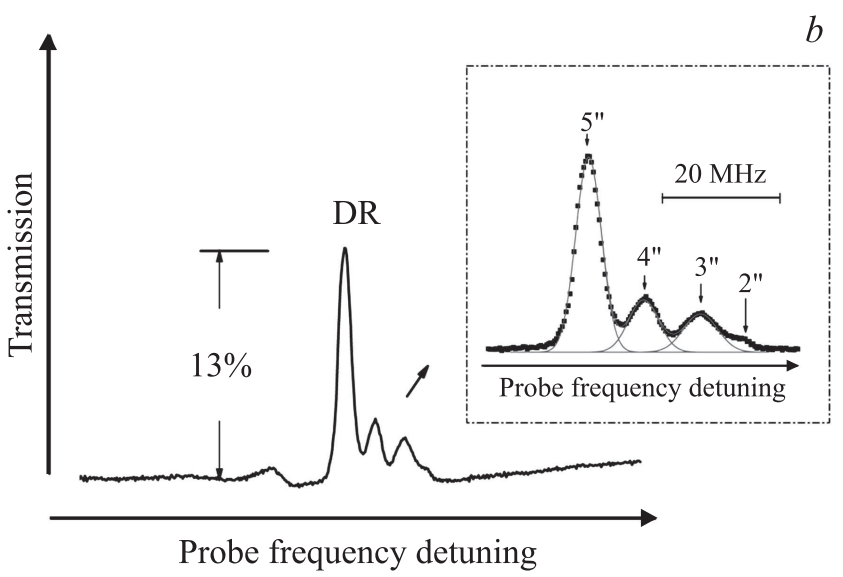

Рис. 3. ${ }^{85} \mathrm{Rb}, \mathrm{D}_{2}$-линия, толщина ячейки $L=4 \mathrm{~mm}$, $\Xi$-система. (a) Кривая 1 - спектр пропускания пробной волны, содержащий DR, температура ячейки $85^{\circ} \mathrm{C}$, кривая $2-$ то же при температуре ячейки $145^{\circ} \mathrm{C}$; на вставке показан профиль DR, аппроксимированный лоренцевой кривой с шириной на полувысоте 7.5 MHz. Наблюдается 22-кратное сужение спектра DR. Нижняя кривая - Reference - реперный спектр, полученный методом насыщенного поглощения (НП); в реперную ячейку попадает также часть связывающего излучения, что приводит к некоторой модификации амплитуд в спектре НП. (b) Спектр пропускания пробной волны, содержащий DR. Температура ячейки $90^{\circ} \mathrm{C}$, мощности пробной и связывающей волн: $1 \mu \mathrm{W}$ и $4 \mathrm{~mW}$ соответственно, контраст DR 13\%. На вставке аппроксимированные гауссовыми кривыми профили DR, переходы $5 P_{3 / 2}-5 D_{5 / 2}\left(F_{g}=2,3,4^{\prime} \rightarrow F_{e}=2^{\prime \prime}, 3^{\prime \prime}, 4^{\prime \prime}, 5^{\prime \prime}\right)$.

формировании. Важно отметить, что спектральная ширина DR в этом случае составляет $5 \mathrm{MHz}$ (рис. $3, b$ ), поэтому сверхтонкая структура уровня $5 D_{5 / 2}$ полностью спектрально разрешается. Измеренные частотные интервалы сверхтонкой структуры уровня $5 D_{5 / 2}$, а именно $\Delta\left(5^{\prime \prime}-4^{\prime \prime}\right), \Delta\left(4^{\prime \prime}-3^{\prime \prime}\right)$ и $\Delta\left(3^{\prime \prime}-2^{\prime \prime}\right)$ составляют 9.5, 9 и $7.5 \mathrm{MHz}$ соответственно, что согласуется с результатами работы [21].

В работе [22] для $\Lambda$-системы было показано 4-кратное спектральное сужение спектра DR, и было приведено 
выражение для эффекта сужения:

$$
\gamma_{\mathrm{DR}}=\frac{\Omega_{c}^{2}}{\sqrt{\gamma_{12} \gamma_{2}}} \frac{1}{\sqrt{\sigma N L}},
$$

где $\sigma=3 \lambda^{2}(2 \pi)^{-1}-$ сечение резонансного поглощения, $N$ - концентрация атомов, $L-$ длина ячейки, $\Omega_{c}$ - частота Раби, $\gamma_{2}$ и $\gamma_{12}$ - скорости распада промежуточного состояния и когерентности между промежуточным и основным состояниями соответственно. Для параметров эксперимента $L=0.4 \mathrm{~cm}, \lambda=780 \mathrm{~nm}$, $N_{\mathrm{Rb}}=10^{16} \mathrm{~cm}^{-3}$ величина $(\sigma N L)^{1 / 2}$ может достигать $3 \times 10^{3}$. С целью выявления, до каких малых толщин имеет место эффект сужения спектра DR, были использованы ячейки малой толщины.

\subsection{2. Толщина столба паров $L=2.5 \lambda=1950 \mathrm{~nm}$}

Кривая 1 на рис. 4, a показывает спектр пропускания пробной волны, который содержит два DR для атомов ${ }^{85} \mathrm{Rb}$ и ${ }^{87} \mathrm{Rb}$ при толщине $L=2.5 \lambda=1950 \mathrm{~nm}$ и температуре ячейки $120^{\circ} \mathrm{C}$ (плотность атомов $N_{\mathrm{Rb}} \sim 2 \cdot 10^{13} \mathrm{~cm}^{-3}$ ), мощности пробной и связывающей волн составляли $0.4 \mu \mathrm{W}$ и $250 \mathrm{~mW}$ соответственно. При этом достигается контраст DR 95\% как для атомов ${ }^{85} \mathrm{Rb}$, так и для атомов ${ }^{87} \mathrm{Rb}$. Спектральная ширина DR для ${ }^{85} \mathrm{Rb}$ на полувысоте $\sim 240 \mathrm{MHz}$. Кривая 2 на рис. 4, $a$ показывает спектр пробной волны для атомов ${ }^{85} \mathrm{Rb}$ и ${ }^{87} \mathrm{Rb}$, который содержит два DR, температура ячейки $255^{\circ} \mathrm{C}$ (плотность $\left.N_{\mathrm{Rb}} \sim 5 \cdot 10^{15} \mathrm{~cm}^{-3}\right)$. Спектральная ширина DR для ${ }^{85} \mathrm{Rb}$ на полувысоте $\sim 100 \mathrm{MHz}$, т.е. имеет место 2.4кратное сужение спектра DR. На рис. $4, b$ приведена зависимость спектральной ширины DR при изменении плотности $N_{\mathrm{Rb}}$ в интервале $10^{13}-10^{16} \mathrm{~cm}^{-3}$, которая хорошо аппроксимируется эмпирической формулой $\gamma_{\mathrm{DR}}[\mathrm{MHz}]=15 \mathrm{MHz}+1.1 \times 10^{5} \times N^{-0.2}\left[\mathrm{~cm}^{-3}\right]$.

На рис. 5 приведены спектры пропускания пробного излучения при $L=1950 \mathrm{~nm}$ в отмеченной выше $\Xi$-системе атомов ${ }^{85} \mathrm{Rb}$ и ${ }^{87} \mathrm{Rb}$ при возрастании мощности связывающего излучения $P_{C}$. Кривые, отмеченные цифрами $1-5$, соответствуют мощностям $P_{C}=5,10,70,190$ и $250 \mathrm{~mW}$ соответственно, мощность $P_{P}=0.4 \mu \mathrm{W}$, температура $124^{\circ} \mathrm{C}$. Как и ожидалось, с увеличением $P_{C}$ происходит увеличение контраста DR до $\sim 100 \%$, а также спектральное уширение темного резонанса. Нижняя кривая - спектр пропускания пробного излучения при отсутствии связывающего излучения. Как было показано в работе [23], при толщине столба паров атомов $L=2.5 \lambda$ спектр пропускания демонстрирует эффект сужения Дике, что проявляется в модификации допплеровски уширенного спектра пропускания. На рис. 6, $a$ приведена зависимость контраста DR, определенная по спектрам, приведенным на рис. 5, при изменении мощности связывающего излучения $P_{C}$, которая хорошо аппроксимируется эмпирической формулой $C(\%)=29 \times P^{0.22}[\mathrm{~mW}]$. На рис. $6, b$
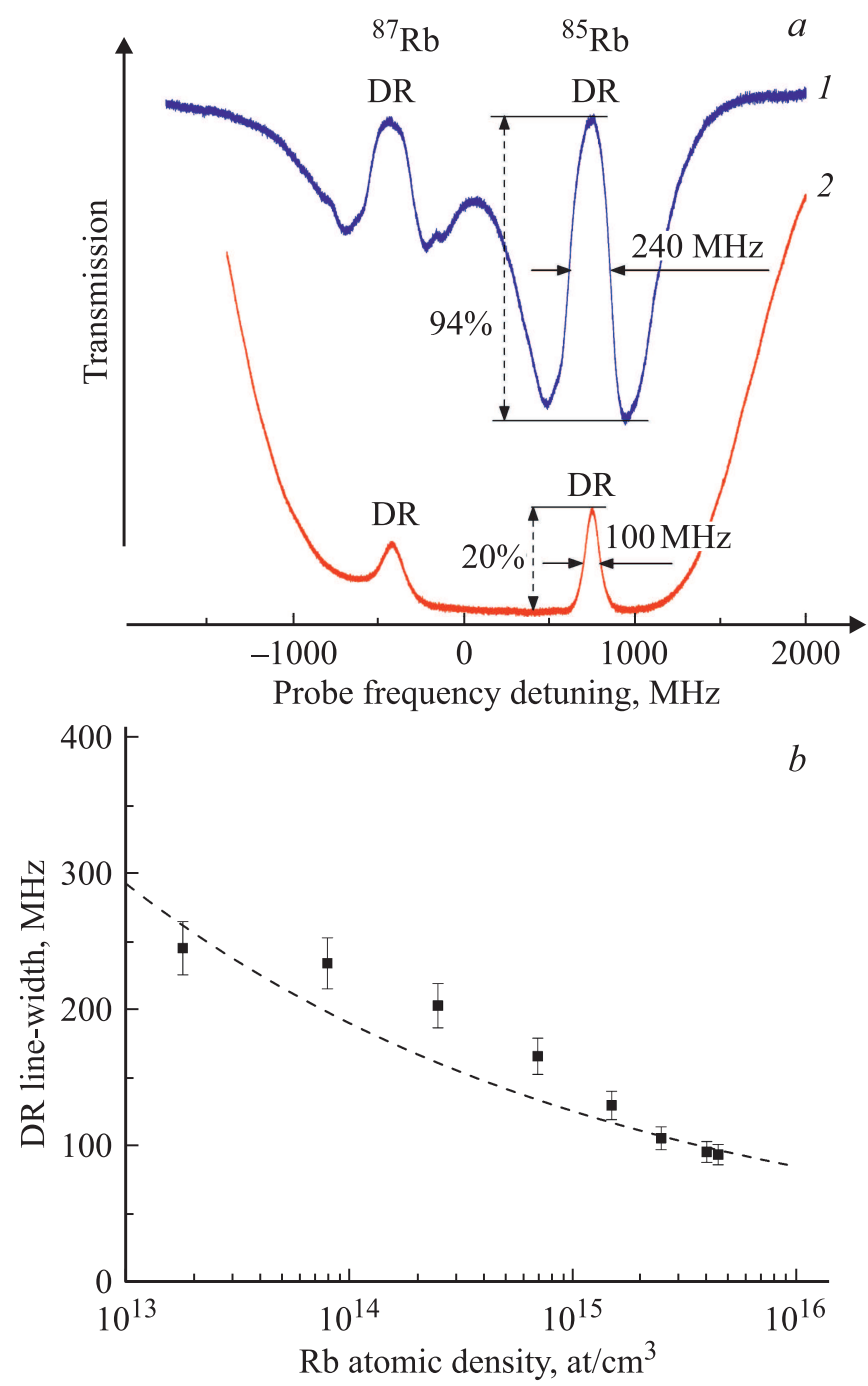

Рис. 4. Толщина ячейки $L=2.5 \lambda=1950 \mathrm{~nm}$. (a) Кривая 1 спектр пропускания пробной волны, который содержит два DR для атомов ${ }^{85} \mathrm{Rb}$ и ${ }^{87} \mathrm{Rb}$ при толщине $L=2.5 \lambda=1950 \mathrm{~nm}$ и температуре ячейки $120^{\circ} \mathrm{C}$, спектральная ширина DR для ${ }^{85} \mathrm{Rb} \sim 240 \mathrm{MHz}$, кривая 2 - спектр пропускания пробной волны, который содержит два DR при температуре ячейки $255^{\circ} \mathrm{C}$, спектральная ширина DR $\sim 100 \mathrm{MHz} ;(b)$ Зависимость спектральной ширины DR от плотности атомов $N_{\mathrm{Rb}}$ в интервале $10^{13}-10^{16} \mathrm{~cm}^{-3}$. Ошибка измерения $\sim 8 \%$.

приведена зависимость спектральной ширины DR при изменении мощности связывающего излучения $P_{C}$, которая хорошо аппроксимируется эмпирической формулой $\gamma_{\mathrm{DR}}[\mathrm{MHz}]=14 \times P^{0.5}[\mathrm{~mW}]$. Поскольку частота Раби $\Omega_{C} \sim P^{0.5}$, то имеем зависимость для ширины $\gamma_{\mathrm{DR}} \sim \Omega_{C}$. На линейную зависимость ширины $\gamma_{\mathrm{DR}}$ от частоты Раби указано в работе [24].

\subsection{3. Толщина столба $L=\lambda=780 \mathrm{~nm}$}

Была исследована зависимость спектральной ширины $\mathrm{DR}$ от плотности атомов $\mathrm{Rb} \Xi$-системе при толщине ячейки $L=\lambda=780 \mathrm{~nm}$. Особенности поведения DR при 


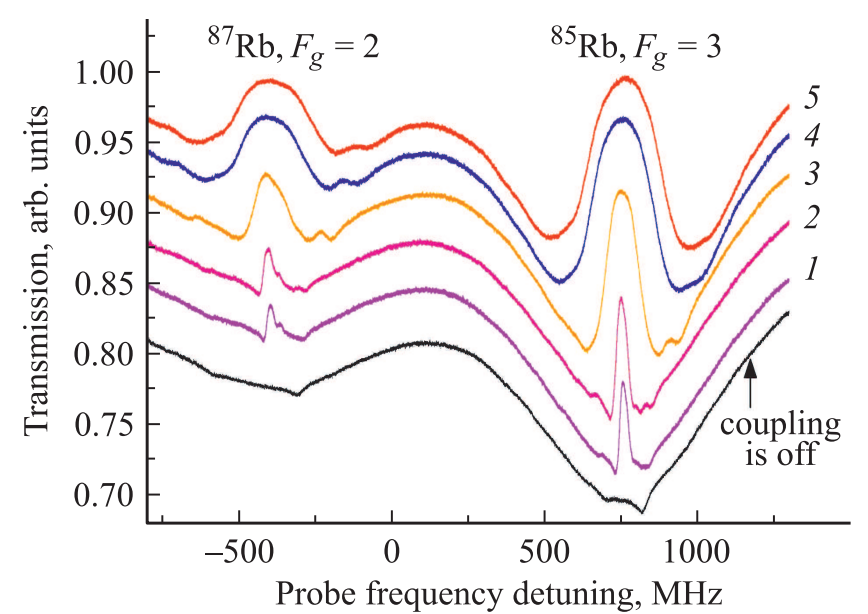

Рис. 5. Толщина ячейки $L=2.5 \lambda=1950 \mathrm{~nm}$. Спектры пропускания пробного излучения в $\Xi$-системах атомов ${ }^{85} \mathrm{Rb}$ и ${ }^{87} \mathrm{Rb}$ при возрастании мощности связывающего излучения (снизу вверх) $P_{C}: 5,10,70,190$ и $250 \mathrm{~mW}$, мощность $P_{P}=0.4 \mu \mathrm{W}$, температура $124^{\circ} \mathrm{C}$. Нижняя кривая (coupling is off) - спектр пропускания пробного излучения при отсутствии связывающего излучения.

такой толщине приведены в работе [25]. Однако исследования поведения спектрального сужения DR от плотности атомов $\mathrm{Rb}$ в ней не проводились. На рис. 7 приведена зависимость спектральной ширины DR от плотности атомов ${ }^{85} \mathrm{Rb}$. Частота пробного излучения $v_{P}$ сканируется по переходам $F_{g}=3 \rightarrow F_{e}=2^{\prime}, 3^{\prime}, 4^{\prime} D_{2}$ линии атома ${ }^{85} \mathrm{Rb}$, а частота $v_{C}$ находится в резонансе с переходом $5 P_{3 / 2}-5 D_{5 / 2} \quad\left(F_{e}=4^{\prime} \rightarrow F_{e}=3^{\prime \prime}, 4^{\prime \prime}, 5^{\prime \prime}\right)$. Мощности пробной и связывающей волн составляли $1 \mu \mathrm{W}$ и $180 \mathrm{~mW}$ соответственно. Как видно из кривой на рис. 7, спектральная ширины DR практически не меняется вплоть до $N \sim 10^{15} \mathrm{~cm}^{-3}$; небольшое $20 \%$ сужение наблюдается только при больших концентрациях атомов $N \sim 4 \cdot 10^{15} \mathrm{~cm}^{-3}$

\subsection{4. Толщина ячейки $L=\lambda / 2=390 \mathrm{~nm}$}

Для исследования зависимости ширины DR от плотности атомов $\mathrm{Rb}$ в $\Xi$-системе при толщине ячейки $L=\lambda / 2=390 \mathrm{~nm}$ была использована техника синхронного детектирования, что существенно улучшает отношение сигнал/шум [10,25]. Это техническое усложнение необходимо, поскольку амплитуда DR при такой малой толщине мала. Мощности пробной и связывающей волн составляли $0.4 \mu \mathrm{W}$ и $125 \mathrm{~mW}$ соответственно. Кривая 1 на рис. 8, $a$ показывает спектр пробной волны, который содержит два DR для атомов ${ }^{85} \mathrm{Rb}$ и ${ }^{87} \mathrm{Rb}$ при температуре ячейки $142^{\circ} \mathrm{C}$ (плотность атомов $N_{\mathrm{Rb}} \sim 5 \cdot 10^{13} \mathrm{~cm}^{-3}$ ), спектральная ширина DR $\left({ }^{85} \mathrm{Rb}\right) 260 \mathrm{MHz}$. Кривая 2 на рис. 8 , $а$ показывает спектр пропускания пробной волны, который содержит два DR для атомов ${ }^{85} \mathrm{Rb}$ и ${ }^{87} \mathrm{Rb}$ при температуре ячейки $300^{\circ} \mathrm{C}$ (плотность атомов $N_{\mathrm{Rb}} \sim 2 \cdot 10^{16} \mathrm{~cm}^{-3}$ ), спектральная
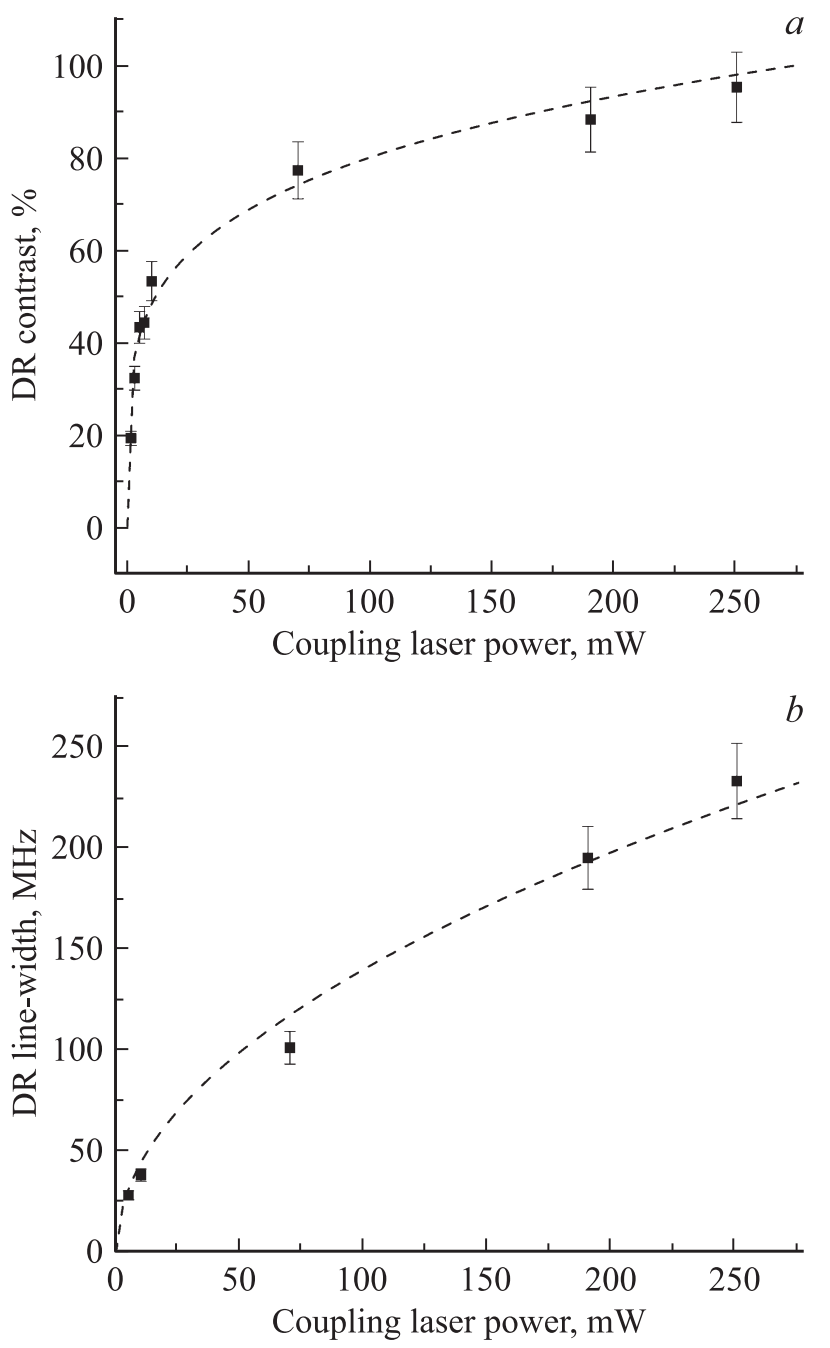

Рис. 6. Толщина ячейки $L=2.5 \lambda=1950 \mathrm{~nm}$. (a) зависимость контраста DR при изменении мощности связывающего излучения $P_{C} ;(b)$ зависимость спектральной ширины DR при изменении мощности связывающего излучения $P_{C}$.

ширина DR $450 \mathrm{MHz}$, т. е. имеет место уширение спектра DR. На рис. 8, $b$ приведена зависимость спектральной ширины DR при изменении плотности $N_{\mathrm{Rb}}$ в интервале $10^{13}-10^{16} \mathrm{~cm}^{-3}$, которая хорошо аппроксимируется эмпирической формулой $\gamma[\mathrm{MHz}]=11 \times N^{0.1}\left[\mathrm{~cm}^{-3}\right]$. Слабая зависимость ширины спектра от плотности паров при толщине ячейки $L=\lambda / 2=390 \mathrm{~nm}$ объясняется частыми столкновениями атомов с окнами ячейки.

\section{3. Обсуждение}

Впервые эффект сужения спектра DR был продемонстрирован в известной работе [22] с использованием $\Lambda$ системы. Для регистрации сужения использовался процесс когерентного пленения населенности, для которого мощности пробного и связывающего излучений должны быть достаточно малы, а оба излучения должны быть когерентно связаны. В этом случае спектральная ширина 


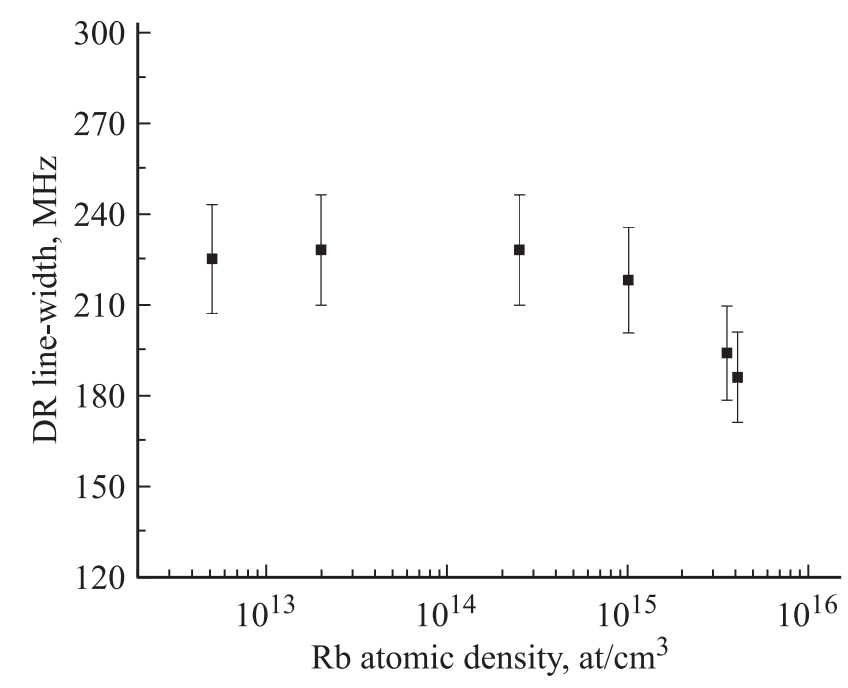

Рис. 7. Толщина ячейки $L=\lambda=780 \mathrm{~nm}$, зависимость спектральной ширины DR от плотности атомов $N_{\mathrm{Rb}}$ в интервале $10^{13}-10^{16} \mathrm{~cm}^{-3}$.

DR составляла несколько десятков $\mathrm{kHz}$ и было продемонстрировано 4-кратное спектральное сужение [22]. O наблюдении эффекта сужения спектра DR с использованием процесса ЭИП в случае, когда используются два независимых лазера (с мощностями в несколько $\mathrm{mW}$, a спектральная ширина DR составляет несколько $\mathrm{MHz}$ ) с использованием $\Lambda$-системы, в литературе не сообщалось. В настоящей работе показано, что эффект сужения спектра DR в $\Xi$-системе не так критичен к параметрам эксперимента, как это имеет место в $\Lambda$-системе, а также зарегистрировано 22-кратное сужение, что значительно превышает сужение в $\Lambda$-системе. Преимуществом $\Xi$ системы является слабое поглощение связывающего излучения на втором переходе $(5 P-5 D)$ даже в случае больших плотностей атомов, в то время как в $\Lambda$-системе имеет место большое поглощение связывающего излучения с основного заселенного уровня. К недостаткам $\Xi$-системы следует отнести существенное уширение конечного уровня (в нашем случае $5 D$ ) в случае больших плотностей атомов основного или буферного газа [26], в то время как в $\Lambda$-системе наличие буферного газа практически не уширяет нижние уровни, а увеличение пролетного времени атома через лазерные пучки позволяет формировать DR с шириной в несколько $\mathrm{Hz}$ [11]. В формуле (1) предполагается, что частота Раби для связывающего излучения $\Omega_{C}$ меньше естественных ширин атомных уровней, в случае же больших величин $\gamma_{\mathrm{DR}} \sim \Omega_{c}$ [24] хорошо согласуется с кривой на рис. $6, b$. Зависимость $\gamma_{\mathrm{DR}}$ от плотности атомарных паров $N^{-1 / 2}$ хорошо согласуется при толщинах столба паров $L \sim 1 \mathrm{~cm}$, однако при малых толщинах в несколько микрометров зависимость значительно слабее, а в случае малых толщин $L<\lambda$ вместо спектрального сужения DR имеет место уширение спектра. В случае малых толщин ячейки частые столкновения атомов со стенками ячейки
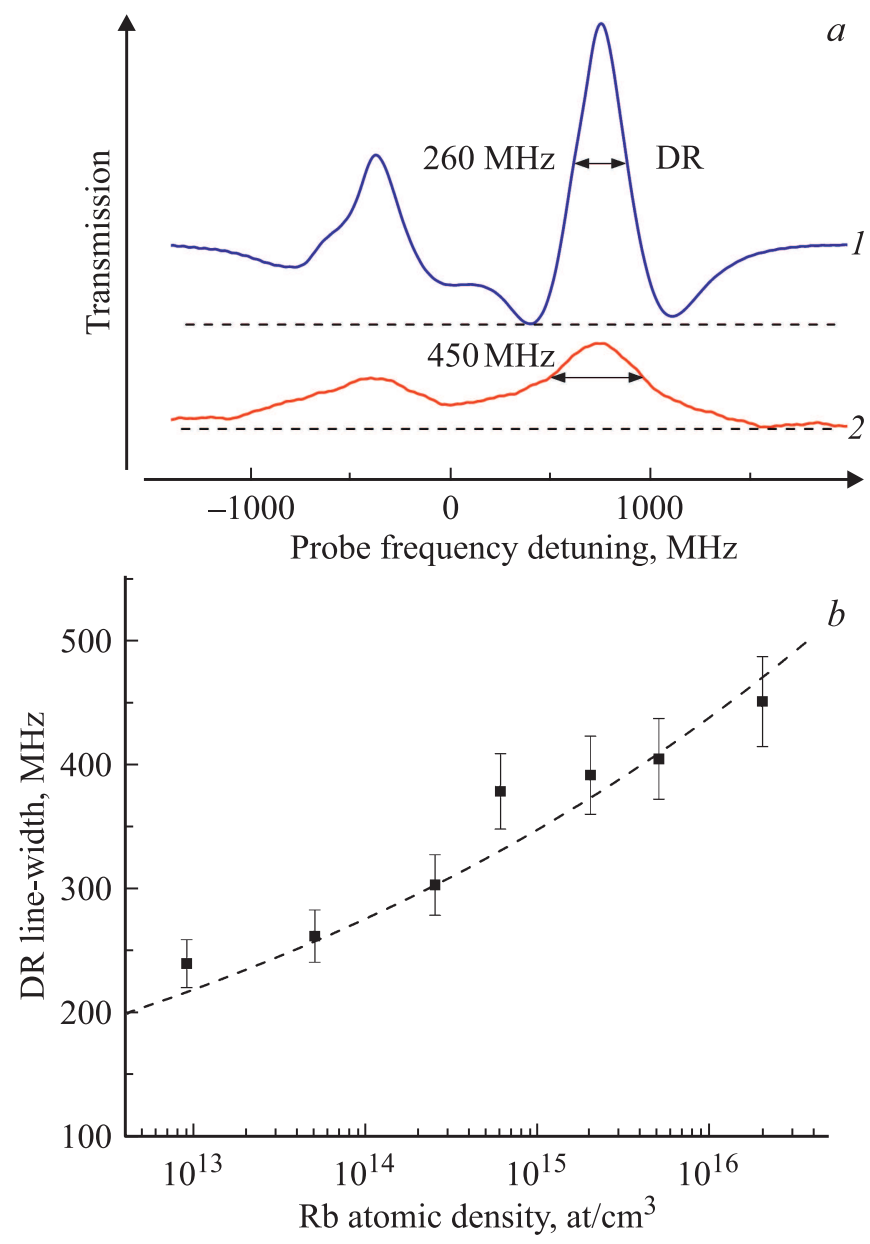

Рис. 8. Толщина ячейки $L=2.5 \lambda=390 \mathrm{~nm},(a)$ кривая 1 спектр пропускания пробного излучения, содержащий два $\mathrm{DR}$ для атомов ${ }^{85} \mathrm{Rb}$ и ${ }^{87} \mathrm{Rb}$, температура ячейки $142^{\circ} \mathrm{C}$, кривая 2 - то же при температуре ячейки $300^{\circ} \mathrm{C} ;(b)$ зависимость спектральной ширины DR от плотности атомов $N_{\mathrm{Rb}}$ в интервале $10^{13}-10^{16} \mathrm{~cm}^{-3}$.

становятся доминирующей причиной уширения, которая подавляет эффект сужения, обусловленный большой плотностью атомов, и приводит к уширению спектра DR. В этом случае ширину DR можно представить как: $\gamma_{\mathrm{DR}} \approx \gamma_{13}+f\left(\Omega_{C}\right)$, где $\gamma_{13}-$ полная скорость дефазировки когерентности, которая обусловлена различного рода уширениями начального $\left(5 S_{1 / 2}\right)$ и конечного $\left(5 D_{5 / 2}\right)$ уровней, в частности, столкновениями атомов со стенками ячейки.

Важно отметить следующее. На рис. $3, b$ мощность связывающего излучения составляет $4 \mathrm{~mW}$. Поэтому, учитывая, что излучение фокусируется в пятно размером $d \approx 30 \mu \mathrm{m}$, для интенсивности получаем $I \sim 0.7 \mathrm{~kW} / \mathrm{cm}^{2}$, и для частоты Раби имеем: $\Omega_{c} / 2 \pi=\gamma_{N}(I / 8)^{1 / 2} \approx 200 \mathrm{MHz}$, где $I-$ интенсивность излучения лазера в $\mathrm{mW} / \mathrm{cm}^{2}$, а $\gamma_{N}-$ естественная ширина возбужденного уровня $5 D_{5 / 2}$ [27]. При таких больших величинах частоты Раби регистрируемая небольшая спектральная ширина $\gamma_{\mathrm{DR}} \approx 5 \mathrm{MHz}$ является несколь- 
ко неожиданной, поскольку оценочные выражения для $\gamma_{\mathrm{DR}} \sim \Omega_{c}^{2} / \gamma_{N}$ [3] предсказывают ширину $\gamma_{\mathrm{DR}}$ на много порядков больше. Кроме того, температура ячейки, а следовательно, и плотность $N_{\mathrm{Rb}}$ недостаточно высока, чтобы имело место вышеотмеченное сужение спектра при больших $N_{\mathrm{Rb}}$. Единственной работой, с которой можно проводить сравнение, является работа [28], в которой при частоте Раби $\Omega_{c} \sim 40 \mathrm{MHz}$ формируемый $\mathrm{DR}$ в парах атомов $\mathrm{Rb}$ имел спектральную ширину меньше естественной. Авторы [28] приводят расчетные кривые, в которых учет допплеровской ширины контура линии поглощения обусловливает сильное сужение $\gamma_{\mathrm{DR}}$. Тем не менее физическое объяснение не может считаться удовлетворительным для $\Xi$-системы, поскольку, как отмечено во введении, остаточная допплеровская ширина в нашем случае $\delta D \approx 2 \mathrm{MHz}$.

Заметим, что система уровней, приведенная на рис. 1 , интересна и тем, что в поле излучений с длинами волн 780 и $776 \mathrm{~nm}$ формируется интенсивное излучение с длиной волны $420 \mathrm{~nm}$ при каскадном переходе $5 D_{5 / 2}-6 P_{3 / 2}$ и далее $6 P_{3 / 2}-5 S_{1 / 2}[29,30]$.

\section{Заключение}

Экспериментально исследован эффект спектрального сужения темного резонанса DR в эффекте электромагнитно-индуцированной прозрачности в $\Xi$ системе в атомарных парах $\mathrm{Rb}$ в системе уровней $5 S_{1 / 2}-5 P_{3 / 2}-5 D_{5 / 2}$. Исследована спектральная ширина $\gamma_{\mathrm{DR}}$ в зависимости от плотности паров атомов и толщины $L$ спектроскопической ячейки, в которой находились пары атомов рубидия. Толщина $L$ варьировалась в интервале от $390 \mathrm{~nm}$ до $4 \mathrm{~mm}$, плотность атомов $N$ варьировалась в интервале $10^{13}-10^{16} \mathrm{~cm}^{-3}$. Использовались сильное связывающее $\left(P_{c}=100-250 \mathrm{~mW}\right)$ и слабое пробное $\left(P_{P}=0.1-6 \mu \mathrm{W}\right)$ излучения, диаметр лазерных пучков составлял $30 \mu \mathrm{m}$. Максимальный эффект, а именно 22кратное спектральное сужение DR достигалось в ячейке с длиной $L=4 \mathrm{~mm}$ при концентрации атомов рубидия $N_{\mathrm{Rb}} \sim 6 \cdot 10^{13} \mathrm{~cm}^{-3}$.

При использовании связывающего излучения малой мощности $4 \mathrm{~mW}$ спектральная ширина DR составляла $5 \mathrm{MHz}$, что позволило полностью спектрально разрешить сверхтонкую структуру уровня $5 D_{5 / 2}$. Нам представляется, что оптимальной толщиной для эффективного наблюдения спектрального сужения DR является $L \sim 400-500 \mu \mathrm{m}$. В этом случае тот же DR контраст 4\% (рис. 3,a) можно получить при большей плотности $N_{\mathrm{Rb}} \sim 6 \cdot 10^{14} \mathrm{~cm}^{-3}$, а величина $(\sigma N L)^{1 / 2}$, которая характеризует эффект спектрального сужения (1), может достигать $10^{4}$.

С уменьшением толщины $L$ эффект сужения становится слабее: так, при $L=1950 \mathrm{~nm}$ происходило всего 2.4-кратное спектральное сужение DR. Спектральное сужение DR практически отсутствовало при $L=780 \mathrm{~nm}$, а при дальнейшем уменьшении до $L=390 \mathrm{~nm}$ с увеличением плотности паров атомов начинало происходить спектральное уширение DR. Приведено объяснение эффекта спектрального сужения и уширения DR. Практически во всех случаях при умеренных плотностях атомов и больших интенсивностях связывающего излучения достигался $\sim 100 \%$ контраст DR. В работе показано, что эффект сужения спектра DR с использованием $\Xi$ системы не так критичен к параметрам эксперимента, как это имеет место в $\Lambda$-системе атомов $\mathrm{Rb}$; также зарегистрированное 22-кратное сужение значительно превышает 4-кратное сужение, зарегистрированное в $\Lambda$ системе в работе [22]. Приведено выражение для описания эффекта спектрального сужения темного резонанса DR для $\Lambda$-системы, которое качественно хорошо объясняет эффект спектрального сужения DR и в $\Xi$-системе. Проведено сравнение преимуществ и недостатков $\Lambda$ - и $\Xi$-систем для формирования DR.

Приведенные результаты важны для практических приложений, поскольку позволяют значительно уменьшить размеры используемых атомарных ячеек, что важно для миниатюризации устройств при применении DR.

\section{Благодарности}

Авторы благодарят Дж. Кевени за полезные обсуждения.

\section{Финансирование работы}

Авторы благодарят ГК МОН РА за финансовую поддержку, проект № 18T-1С018. А. Саргсян также благодарит ГК МОН РА за финансовую поддержку, проект для молодых ученых № 19YR-1C017. Работа T.A. Вартаняна была выполнена при государственной финансовой поддержке ведущих университетов Российской Федерации (субсидия 08-08).

\section{Конфликт интересов}

Авторы заявляют, что у них нет конфликта интересов.

\section{Список литературы}

[1] Агапьев Б.Д., Горный М.В., Матисов Б.Г., Рождественский Ю.В. // УФН. 1993. Т. 163. С. 1.

[2] Gea-Banacloche J., Li Y.-Q., Jin S.-Z., Min Xiao. // Phys. Rev. A. 1995. V. 51. P. 576.

[3] Wynands R., Nagel A. // Appl. Phys. B. 1999. V. 68. P. 1.

[4] Fleischhauer M., Imamoglu A., Marangos J.P. // Rev. Mod. Phys. 2005. V. 77. P. 633.

[5] Sargsyan A., Sarkisyan D., Papoyan A. // Phys. Rev. A. 2006. V. 73. P. 033803.

[6] Knappe S., Hollberg L., Kitching J. // Opt. Lett. 2004. V. 29. P. 388.

[7] Kitching J. // Appl. Phys. Rev. 2018. V. 5. P. 031302.

[8] Mohapatra A.K., Jackson T.R., Adams C.S. // Phys. Rev. Lett. 2007. V. 98. P. 113003. 
[9] Bason M.G., Tanasittikosol M., Sargsyan A., Mohapatra A.K., Sarkisyan D., Potvliege R.M., Adams C.S. // New J. Phys. 2010. V. 12. P. 065015.

[10] Саргсян А., Бейсон М., Саркисян Д., Мохапатра А., Адамс Ч. // Опт. и спектр. 2010. Т. 109. С. 581.

[11] Brandt S., Nagel A., Wynands R., Meschede D. // Phys. Rev. A. 1997. V. 56. P. R1063.

[12] Laskar A.W., Singh N., Adhikary P., Mukherjee A., Ghosh S. // Optica. 2018. V. 5. P. 1462.

[13] Moon H.S., Noh H.-R. // Opt. Express. 2013. V. 21. P. 7447.

[14] Moseley R.R., Shepherd S., Fulton D.J., Sinclair B.D., Dunn M.H. // Optics Commun. 1995. V. 119. P. 61.

[15] Moon H.S., Noh H.-R. // J. Opt. Soc. Am. B. 2012. V. 29. P. 1557.

[16] Perrella C., Light P.S., Anstie J.D., Stace T.M., Benabid F., Luiten A.N. // Phys. Rev. A. 2013. V. 87. P. 013818.

[17] Sarkisyan D., Bloch D., Papoyan A., Ducloy M. // Opt. Commun. 2001. V. 200. P. 201.

[18] Саргсян А., Амирян А., Леруа К., Вартанян Т., Саркисян Д. // Опт. и спектр. 2017. Т. 123. С. 124.

[19] Demtroder W. Laser Spectroscopy: Basic Concepts and Instrumentation, NY:: Springer, 2004.

[20] Keaveney J., Sargsyan A., Krohn U., Sarkisyan D., Papoyan A., Adams C.S. // J. Phys. B. 2014. V. 47. P. 075002.

[21] Olson A.J., Carlson E.J., Mayer S.K. // Am. J. Phys. 2006. V. 74. P. 218.

[22] Lukin M.D., Fleischhauer M., Zibrov A.S., Robinson H.G., Velichansky V.L., Hollberg L., Scully M.O. // Phys. Rev. Lett. 1997. V. 79. P. 2959.

[23] Cartaleva S., Saltiel S., Sargsyan A., Sarkisyan D., Slavov D., Todorov P., Vaseva K. // J. Opt. Soc. Am. B. 2009. V. 26. P. 1999.

[24] Yang L., Zhang L., Guo Q., Fu G. // J. Luminescence. 2007. V. 122. P. 552.

[25] Саркисян Д., Саргсян А., Кевени Дж., Адамс Ч. // ЖЭТФ. 2014. T. 146. C. 13.

[26] Sargsyan A., Sarkisyan D., Krohn U., Keaveney J., Adams Ch. // Phys. Rev. A. 2010. V. 82. P. 045806.

[27] Krmpot A.J., Mijailovic M.M., Panic B.M., Lukic D.V., Kovacevic A.G., Pantelic D.V., Jelenkovic B.M. // Opt. Express. 2005. V. 13. P. 1448.

[28] Iftiquar S.M., Karve G.R., Natarajan V. // Phys. Rev. A. 2008. V. 77. P. 063807.

[29] Akulshin A.M., McLean R.J., Sidorov A.I., Hannaford P. // Opt. Express. 2009. V. 17. P. 22861.

[30] Akulshin A.M., Budker D., McLean R.J. // JOSA B. 2017. V. 34. P. 1016. 